\title{
De novo presenilin 1 mutations are rare in clinically sporadic, early onset Alzheimer's disease cases
}

\author{
Laboratoire de \\ Génétique \\ Moléculaire, CHU de \\ Rouen, 76031 Rouen, \\ France \\ C Dumanchin \\ D Campion \\ C Martin \\ $\mathrm{V}$ Moreau \\ T Frebourg
}

IFRMP23, 76821

Mont-Saint-Aignan

Cedex, France

C Dumanchin

T Frebourg

INSERM U289, 75013

Paris, France

A Brice

Y Agid

Département de

Recherche du CHSR,

Université de Rouen,

76300 Sotteville les

Rouen, France

D Campion

Clinique

Neurologique, CHU de

Rouen, 76031 Rouen,

France

D Hannequin

INSERM U358,

Hôpital St Louis, 75010

Paris, France

M Martinez

INSERM U155,

Chateau de

Longchamp, 75016

Paris, France

F Clerget-Darpoux

${ }^{\star}$ Catherine

Thomas-Anterion,

Michèle Puel, Bruno

Dubois, Olivier Moreaud.

Correspondence to:

Dr Frebourg.

Received 14 July 1997

Revised version accepted for publication 13 January 1998

Table 1 Characteristics of the EOAD patients

\begin{tabular}{lllll}
\hline Patient & Sex & Age of onset $(y)$ & PS1 mutation & ApoE genotype \\
\hline ALZ 004 & M & 50 & $-\star$ & $\varepsilon 3 / \varepsilon 4$ \\
ALZ 021 & F & 45 & - & $\varepsilon 3 / \varepsilon 3$ \\
LIL 002 & F & 50 & - & $\varepsilon 3 / \varepsilon 3$ \\
SAL 599 & F & 45 & - & $\varepsilon 3 / \varepsilon 3$ \\
SAL 590 & F & 49 & - & $\varepsilon 3 / \varepsilon 4$ \\
CRN 008 & M & 43 & - & $\varepsilon 3 / \varepsilon 3$ \\
CRN 001 & F & 41 & - & $\varepsilon 3 / \varepsilon 3$ \\
REN 001 & F & 50 & - & $\varepsilon 3 / \varepsilon 3$ \\
ROU 004 & M & 41 & - & $\varepsilon 3 / \varepsilon 3$ \\
ROU 060 & M & 50 & - & $\varepsilon 3 / \varepsilon 3$ \\
ALZ 034 & F & 37 & - & $\varepsilon 3 / \varepsilon 3$ \\
CAE 016 & F & 50 & & $\varepsilon 3 / \varepsilon 4$ \\
SAL 008 & F & 50 & & \\
\hline
\end{tabular}

^No mutation was detected.

\begin{abstract}
The presenilin 1 (PS1) gene, located on chromosome 14, is the major gene involved in the autosomal dominant forms of early onset Alzheimer's disease (AD). In order to estimate the frequency of de novo PS1 mutations, we have sequenced the PS1 open reading frame in 13 clinically diagnosed patients with no affected relatives, who had developed $A D$ before the age of 50 . In one case with onset at 37 years, we identified a missense mutation resulting in a methionine to lysine substitution at codon 139 of the PS1 gene. This substitution is the fourth identified at the same codon. This study, in agreement with previous reports, suggests that de novo PS1 mutations can occur but at a low frequency.
\end{abstract}

(F Med Genet 1998;35:672-673)

Keywords: Alzheimer's disease; presenilin 1; mutations; sporadic cases

Alzheimer's disease, the most common cause of dementia in the elderly, is inherited in an autosomal dominant fashion in a subset of patients with early onset of the disease. Three genes involved in these autosomal dominant forms of early onset Alzheimer's disease (EOAD) have been identified so far: the amyloid precursor protein (APP) gene located on chromosome $21 \mathrm{q} 21,{ }^{1}$ the presenilin 1 (PS1) gene located on chromosome $14 \mathrm{q} 24.3,{ }^{2}$ and the presenilin 2 (PS2) gene located on chromosome $1 \mathrm{q} 42.1 .^{3-5}$ The PS1 gene is the major locus for familial EOAD, since PS1 mutations have been identified in approximately $30-50 \%$ of the families. ${ }^{6}$ Except for one splicing mutation, ${ }^{7}$ all the PS1 alterations documented so far have been missense mutations. These mutations show remarkable allelic

Cécile Dumanchin, Alexis Brice, Dominique Campion, Didier Hannequin, Cosette Martin, Viviane Moreau, Yves Agid, Maria Martinez, Françoise Clerget-Darpoux, Thierry Frebourg, and the French Alzheimer's Disease Study Group ${ }^{\star}$

heterogeneity since approximately 40 PS 1 amino acid substitutions have been documented in at least 70 families with $\mathrm{EOAD},{ }^{6}$ and we have identified 13 different missense mutations in 15 French families ${ }^{89}$ (unpublished data). In two families, in which we had identified a PS1 mutation, the absence of affected relatives two generations ago led us to speculate that the mutation had occurred de novo. In order to assess the frequency of de novo PS1 mutations, we performed sequencing analysis of the PS1 gene in EOAD patients with no affected relatives.

\section{Subjects and methods}

PATIENTS

This study was performed in 13 unrelated French patients fullfilling the NINCDSADRDA criteria for probable $\mathrm{AD} .^{10}$ These patients were selected according to two criteria:

(1) onset of $\mathrm{AD}$ before the age of 50 years and

(2) being isolated cases as defined by absence of affected sibs, parents, and uncles/aunts. After informed consent was obtained, peripheral blood lymphocytes were collected and Epstein-Barr virus transformed lymphoblastoid cell lines were established. The APOE genotyping of these patients was performed by HhaI restriction fragment analysis. ${ }^{11}$

SCREENING FOR PS1 MUTATIONS

The coding region of the PS1 gene was analysed by reverse transcription-polymerase chain reaction (RT-PCR) and direct sequencing, as previously described ${ }^{8}$ except that the regions corresponding to codons $72-187$ and 164-287 were separately amplified using the $901 F^{2}, 901 \mathrm{R}$ primers (GTC CAC AGC AAC GTT ATA GG) and the $1111 \mathrm{~F}$ (GTG CTA TAA GGT CAT CCA TG) and $1111 \mathrm{R}^{2}$ primers, respectively. To perform dye primer sequencing, we added a $M 13$ reverse sequence (5'-CAGGAAACAGCTATGACC-3') to the 5' end of the sense primers $(901 \mathrm{~F}$ and $1111 \mathrm{~F})$ and a M13-21 sequence (5'-TGTAAAACGACGGCCAGT-3') to the 5' end of the antisense primers (901R and $1111 \mathrm{R}$ ), respectively.

\section{Results}

Among the 13 patients with clinically sporadic EOAD (table 1), we identified in one subject (ALZ034) a heterozygous mutation (ATG $\rightarrow$ AAG) at codon 139 , which is predicted to result in a methionine to lysine substitution (data not shown). This mutation, Met139Lys, removes a $B c l$ restriction site which allowed us to test by restriction fragment analysis, as previously described, ${ }^{8}$ the mother 
I

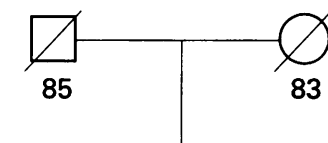

II
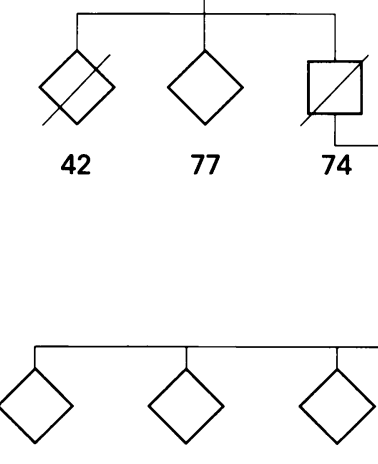

53

49

77

\begin{abstract}
74
\end{abstract}
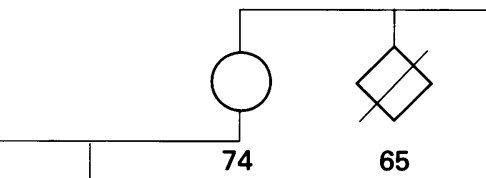

65

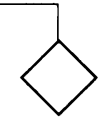

62

III
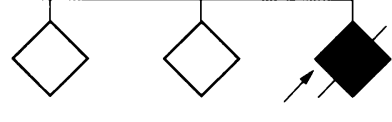

47 (37)

47

44

$+/ 139$

Figure 1 Pedigree of family ALZ034. Filled symbol, affected subject; open symbols, asymptomatic subjects; numbers under the symbols are ages at examination or death. The number in brackets is age of onset of $A D ;+/+$, wild type genotype; +1139 , heterozygous Met 139Lys mutation. The pedigree has been partially encrypted to protect confidentiality of family members. tion analysis of two unaffected first degree relatives of the ALZ034 proband, aged 75 and 50 years, showed that they do not carry the mutation. The father was dead, unaffected by the age of 74, and had no family history of $\mathrm{AD}$ among his sibs or parents (fig 1). Although one PS1 point mutation with incomplete penetrance by the age of 68 has recently been described, ${ }^{12}$ it seems very unlikely from the examination of the pedigree that nonpenetrance is involved in our family. The alternative explanation, assuming that paternity is correct, is that this substitution has occurred de novo. Recently, studies ${ }^{13} 14$ have incidently reported the occurrence of de novo PS1 mutations in isolated EOAD patients. Our study shows that a low proportion of clinically sporadic EOAD cases could be explained by such de novo mutations. We suggest that isolated cases with onset below the age of 50 should be screened for PS1 mutations.

This work was supported by grants from the Institut National de la Santé et de la Recherche Médicale (INSERM, Network 492002), the CNAMTS (Caisse Nationale d'Assurance Maladie des Travailleurs Sociaux, and the MGEN (Mutuelle Générale de l'Education Nationale). C Dumanchin was supported by Le Conseil Régional de Haute-Normandie.

one brother of the proband. No mu was detected among these relatives. In this family, the father was dead and was unaffected by the age of 74 (fig 1 ). Among the 13 patients, the majority $(n=9)$ had the APOE $\varepsilon 3 / \varepsilon 3$ genotype, four had the $\varepsilon 3 / \varepsilon 4$ genotype, and none had the $\varepsilon 4 / \varepsilon 4$ genotype (table 1 ).

\section{Discussion}

We have analysed the PS1 gene in a sample of 13 clinically diagnosed EOAD patients (range of onset ages 37 to 50 years) with no affected relatives. Using a RT-PCR approach, we identified, in one patient who had developed $\mathrm{AD}$ by the age of 37, a new missense mutation of the PS1 gene. RT-PCR has been shown to be an efficient method for detecting PS1 mutations, ${ }^{8}$ but we cannot exclude that in the other patients we have missed mutations located outside the coding region or resulting in mRNA decay. Although we cannot exclude that the Met139Lys substitution corresponds to a rare variation without any biological consequence for PS1 function, we consider that this substitution is probably pathogenic; we did not detect this substitution in more than 50 unrelated subjects, indicating that it is not a common polymorphism. Furthermore, codon 139 corresponds to a hot spot of mutation since three different substitutions (Met139Ile, Met139Thr, Met139Val) have already been described in EOAD families. ${ }^{6}$ Codon 139, in exon 5, is located in one of the two PS1 regions in which most mutations are found ${ }^{6}$ Restric-
1 Goate A, Chartier-Harlin MC, Mullan M, et al. Segregation of a missense mutation in the amyloid precursor protein gene with familial Alzheimer's disease. Nature 1991;349:704-6.

2 Sherrington R, Rogaev EI, Liang Y, et al. Cloning of a gene bearing missense mutations in early-onset familial Alzheimer's disease. Nature 1995;375:754-60.

3 Rogaev EI, Sherrington R, Rogaeva EA, et al. Familial a gene on chromosome 1 related to the Alzheimer's disease type 3 gene. Nature 1995;376:775-8.

4 Levy-Lahad E, Wasco W, Poorkaj P, et al. Candidate gene for the chromosome 1 familial Alzheimer's disease locus. Science 1995;269:973-7.

5 Takano T, Sahara N, Yamanouchi Y, et al. Assignment of Alzheimer's presenilin-2 (PS-2) gene to $1 \mathrm{q} 42.1$ by in situ hybridization. Neurosci Lett 1997;221:205-7.

6 Tanzi RE, Kovacs DM, Kim TW, et al. The gene defects responsible for familial Alzheimer's disease. Neurobiol Dis 1996;3:159-68.

7 Perez-Tur J, Froelich S, Prihar G, et al. A mutation in Alzheimer's disease destroying a splice acceptor site in the presenilin-1 gene. NeuroReport 1995;7:297-301.

8 Campion D, Flaman JM, Brice A, et al. Mutations of the disease. Hum Mol Genet 1996;4:2373-7. lin 1 mutation resulting in a familial Alzheimer disease with an onset age of 29 years. NeuroReport 1996;7:1582-4.

10 McKhann G, Drachman D, Folstein M, et al. Clinical diagnosis of Alzheimer's disease: report of the NINCDSnosis of Alzheimer's disease: report of the NINCDS-
ADRDA work group under the auspices of department of health and human services task force on Alzheimer's disease. Neurology 1984;34:939-44

11 Wenham PR, Price WH, Blundell G. Apolipoprotein E genotyping by one-stage PCR. Lancet 1991;337:1158-9.

12 Rossor MN, Fox NC, Beck J, et al. Incomplete penetrance of familial Alzheimer's disease in a pedigree with a novel presenilin 1 gene mutation. Lancet 1996;347:1560.

13 Tanahashi H, Kawakatsu S, Kaneto $M$, et al. Sequence analysis of presenilin 1 gene mutation in Japanese Alzheimer's disease patients. Neurosci Lett 1996;218:139-41.

14 Sandbrink R, Zhang D, Schaeffer S, et al. Missense mutations of the PS1/S182 gene in German early-onset Alzheimer's disease. Ann Neurol 1996;40:265-6. Alzheimer's disease in kindreds with missense mutations in presenilin 1 gene in families with early-onset Alzheimer's

9 Campion D, Brice A, Dumanchin C, et al. A novel preseni- 